\title{
Morbilidad y Mortalidad en Pacientes con Fracturas de Cadera- Hospital EsSalud Tacna 2003-2007
}

RESPONSABLE: Méd. Luis Coronel Amorin

RESUMEN. Se hizo una revisión retrospectiva de 58 historias clínicas de pacientes geriátricos, con fracturas intertrocantéricas y del cuello del fémur atendidos entre enero del 2003 y diciembre del 2007 , en el Hospital III, Daniel A. Carrión EsSalud, Tacna. El promedio de edad fue de 82 años. Fueron operados 57 de 58 pacientes. Se encontró una tasa de mortalidad de $12,07 \%$, siendo la causa más frecuente la sepsis. (42,84\%). La tasa de complicaciones fue de 41,38\% siendo las complicaciones médicas las más frecuentes que las quirúrgicas. Los factores asociados al desarrollo de complicaciones fueron la presencia del antecedente de diabetes (45\%), demencia senil (41\%). Entre los factores asociados a la mortalidad, tuvimos al ACV $(14,29 \%)$ y la falta de deambulación $(3,92 \%)$.
ABSTRACT It was done a retrospective review of medical records of 58 patients with intertrochanterics fractures and neck of the femur treated at the Hospital Daniel Alcides Carrión of Tacna in the time period January 2003 to December 2007. It was found in the study population a predominance of females in $70.69 \%$, the average age was 82 years. We found a mortality rate of $12.07 \%$, being the most frequent cause sepsis (42.84\%). The complication rate was $41.38 \%$ being the most common medical complications that surgery. Factors associated with the development of complications were the presence of a history of diabetes (45\%), dementia (41\%). Among the risk factors associated with mortality, we had a history of stroke (14\%), and lack of ambulation (3\%) Key Words: Hip fractures, Morbility, Mortality
INTRODUCCIÓN. Las fracturas del extremo proximal del fémur son lesiones devastadoras que afectan más a menudo a la población anciana. En la actualidad existe un aumento dramático en su incidencia debido a la mayor longevidad de la población, sobre todo del sexo femenino. Se trata de un grupo de población, que tiene una elevada morbilidad y mortalidad, ya que la gran mayoría de estos pacientes presentan un estado físico deteriorado.

Se estudiaron las fracturas intertrocantéricas y del cuello del fémur. Determinándose la morbilidad y mortalidad que trae consigo la fractura del extremo proximal del fémur en los pacientes en el Hospital III Daniel Alcides Carrión de Tacna, así como el estado de salud previo del paciente y los factores de la actuación hospitalaria, buscando los factores asociados al aumento de la morbi-mortalidad en esta patología.

\section{MARCOTEÓRICO}

Clasificación de las Fracturas del Extremo Proximal del Fémur

Fracturas Intertrocantéricas del Fémur

La clasificación utilizada en el presente trabajo es la clasificación de Tronzo, la cual se basa en su potencial de reducción y estabilidad.

Tipo I. Estable, incompleta, solo con fractura del trocánter mayor, el trocánter menor permanece intacto. Tipo II. Estable, fractura intertrocantérica no conminuta, con ligero desplazamiento o sin él, pared posterior intacta y un fragmento relativamente pequeño en el trocánter menor. Tipo III. Inestable, es decir, carecen de estabilidad interna, no hay ninguna tabla ósea sólida que encuentre a otro trozo óseo de igual solidez: pared psoterior conminurta, con telescopado del espolón del cuello en el fragmento de la diáfisis. El fragmento del trocánter menor es grande y fractura transversa a través del trocánter mayor. Tipo IV. Inestable, pared posterior conminuta sin telescopado de los dos fragmentos principales, de modo que el espolón del cuello está desplazado fuera de la diáfisis. La mayor parte de la pared posterior se pierde hacia adentro. TipoV. Inestable, fractura intertrocantérica oblicua inversa. El trocánter mayor puede estar unido o no con el fragmento trocantérico, y el fragmento de la diáfisis está desplazado hacia adentro. ${ }^{42}$

Fracturas del cuello femoral

La clasificación de GARDEN la divide en cuatro tipos:

La Garden I es una fractura incompleta o impactada. En esta fractura las trabéculas del cuello inferior están todavía intactas. Las Garden II es una fractura completa $\sin$ el desplazamiento. La radiografía demuestra que las trabéculas se interrumpen por una línea de la fractura por el cuello entero del fémur. Garden III es una fractura completa con desplazamiento parcial. Garden IV Los fragmentos están completamente desplazados sobre la base de su estabilidad mecánica. ${ }^{1,40}$ 


\section{Tratamiento}

En principio, el tratamiento de la fracturas del extremo proximal del fémur debe ser quirúrgico; lo único que contraindicaría la operación sería el mal estado general del paciente, lo que causaría alto riesgo en la anestesia y complicaciones relacionadas con la cirugía, pacientes que no deambulen previo a la fractura del extremo proximal del fémur o con demencia severa pueden ser tratados de forma no quirúrgica.

El tratamiento quirúrgico va dirigido a conseguir la fijación estable que conduzca a una consolidación de la fractura en buena posición y sin deformidad, para permitir una movilización lo más precoz posible y deambulación temprana, teniendo como meta principal el retorno del paciente a su nivel funcional previo a la fractura.

\section{Fracturas intertrocantéricas:}

Existen dos grandes grupos de sistemas de fijación interna habitualmente utilizados para las fracturas intertrocantéricas de fémur: los tornillos deslizantes de compresión del extremo proximal del fémur con placa lateral y la placa acodada $\mathrm{AO}$ de $130^{\circ}$ o la de $95^{\circ}$ han resultado ser los implantes adecuados para el tratamiento de estas fracturas, previa reducción y/o impactación en valgo.

Fracturas del cuello del fémur:

\section{Se usa las siguientes opciones quirúrgicas: $:^{38}$}

- Prótesis parcial de cadera: Austin-Moore, Thompson, bipolar o unipolar.

- Prótesis total del extremo proximal del fémur.

\section{Morbilidad y Mortalidad}

\section{Características principales de estos pacientes}

En su mayoría son personas mayores de 70 años. ${ }^{21} \mathrm{El}$ anciano se rompe más la cadera por ser anciano que por su osteoporosis. Mientras que una desviación estándar de la masa ósea multiplica por tres el riesgo de fractura, una caída lo multiplica hasta por treinta veces en el anciano. La falta de reflejos defensivos le hace caer de lado sobre trocánter y le puede transmitir energía que supere un $30 \%$ su resistencia.

Cuando no aparecen complicaciones la mortalidad se reduce al $15 \%$. La aparición de complicaciones pulmonares, cardiacas o vasculares incrementa la tasa de mortalidad al $38 \%{ }^{43}$

Las complicaciones intrahospitalarias más serias están asociadas a una disminución de la expectativa de vida. Entre ellas tenemos la trombosis venosa, los accidentes cerebro-vasculares, la insuficiencia cardiaca, el infarto de miocardio y la sepsis que estuvieron asociados con el periodo más corto de supervivencia. Una de las complicaciones intrahospitalarias más frecuente es la desorientación o el delirio. Según algunos autores un deterioro del estado mental irá invariablemente unido a una complicación ${ }^{25}$.

La mortalidad que se observaba a los seis meses en aquellos pacientes que presentaron como complicación una neumonía es de un $57 \%$, mientras que para aquellos que desarrollaron una infección urinaria es del $42 \%$.La neumonía es la complicación postoperatoria más común. La movilización precoz, intervención precoz, toilet pulmonar vigorosa y fisioterapia son muy importantes para la reducción de las complicaciones postoperatorias. $^{43}$

Entre los factores que influyen adversamente en la mortalidad se encuentra la edad avanzada, el sexo masculino, la localización intertrocantérica y la poca movilidad previa a la fractura ${ }^{43}$. Otros estudios demostraron que el método de tratamiento podía contribuir a la mortalidad postoperatoria ${ }^{50}$

Factores conocidos que influyen en la mortalidad y morbilidad.

Algunos factores asociados con aumento de mortalidad según AAOS (American Asociation of Ortopedic Surgeons) son: la edad avanzada, las enfermedades graves asociadas, el sexo masculino, el tipo de vida sedentario (en una residencia de ancianos) y la demencia ${ }^{23}$.

\section{Edad y sexo}

Estudios epidemiológicos realizados en décadas anteriores confirmaban como factores de riesgo de primer orden la edad, sexo y el bajo contenido de mineral óseo.

La edad avanzada ha sido reconocida como factor de riesgo. Entre las mujeres la tasa de mortalidad se encuentra más elevada hasta igualarse con la de la población general a los 9 meses mientras que los hombres no igualan la tasa de mortalidad de la población general hasta transcurridos $12-18$ meses ${ }^{23}$.

\section{Condiciones médicas presentes al momento de la fractura de cadera}

La mayoría de los autores coinciden en que el estado general previo de estos pacientes es el principal factor de riesgo para la mortalidad. La mejor valoración del estado general crónico se obtiene contabilizando los diferentes diagnósticos médicos: sin enfermedades previas la mortalidad es del $0 \%$, con 1 ó 2 enfermedades asciende al $14 \%$ y con 3 ó más enfermedades al $24 \%{ }^{25}$.

\section{Riesgo Anestésico}

White en 1987 reflejaba en su artículo que los grados ASA I y II asocian una tasa de mortalidad al año de $8 \%$ y los grados III-V $49 \%$. 
Más tarde Michel (2002) ${ }^{26}$ afirmaba que la mortalidad al año en los pacientes con grados ASA III y IV (peor estado de salud) es mayor que en los grados I y II (sanos o medianamente sanos). Por lo tanto, la mejor valoración del estado general agudo tras la fractura se obtiene mediante el grado ASA (American Society of Anestesia).

\section{Estado mental}

La demencia es el factor aislado más importante determinante de la mortalidad.

Otro factor es la continencia de esfínteres, ya que Bird en 1997 afirmaba que la incontinencia urinaria está fuertemente correlacionada con el estado mental.

\section{Parámetros de tratamiento}

Retraso de la intervención. Tiempo transcurrido desde la fractura a la intervención:

Davis y cols. afirmaban que un retraso en la cirugía de más de 24 horas estaba asociado con un incremento en la tasa de mortalidad durante los 28 días posteriores a la fractura. Burlington y Reno observaron la influencia del retraso de intervención. Kenzora afirmaba que un retraso en la intervención menor de una semana permite la estabilización de los problemas médicos. ${ }^{31}$

Los pacientes intervenidos entre $2^{\circ}$ y $5^{\circ}$ día obtenían una tasa de mortalidad del $6 \%$ y los intervenidos antes de 24 horas obtenían una tasa del $34 \%$ al año. SexsonLehner y White afirmaban que los pacientes relativamente sanos (dos o menos condiciones médicas) cuya intervención se retrasaba más de 24 horas presentaban una tasa de mortalidad aumentada el primer año. Pero encontraban que los que tenían 3 ó más patologías obtuvieron peor tasa de mortalidad cuando la intervención se realizaba dentro de las primeras 24 horas. Por este motivo todos los pacientes que tienen una fractura del extremo proximal del fémur necesitan un día o a lo sumo dos para recuperarse de los cambios fisiológicos adaptativos debidos a la fractura ${ }^{32}$.

\section{Tipo de Anestesia.}

Davis no encontró diferencia en cuanto a la mortalidad a corto o largo plazo entre la anestesia general y la regional. Tampoco en las publicaciones del 2002 se encuentra relación entre el tipo de anestesia y la mortalidad.

\section{Tipo de fractura.}

Roos en la década de los 90 afirmaba en sus artículos que las fracturas intertrocantéricas muestran una tasa de mortalidad a los 30 dias mayor. Michel de forma complementaria publica que las fracturas intracapsulares tienen mejor pronóstico. En el mismo año un autor español, Cuenca Espierrez, encuentra que existe un mayor descenso de niveles de hemoglobina en las fracturas intertrocantéricas que en las intracapsulares, probablemente debido a que en estas últimas el sangrado es menor.

Tipo de tratamiento.

La tasa de mortalidad en los no tratados quirúrgicamente es mayor respecto a los tratados quirúrgicamente (11\%) a los 30 días con una ratio de 1,7. La mortalidad en los no operados es mucho mayor en los que permanecieron encamados $(73 \%)$ respecto a los que se movilizaron precozmente con una ratio $3,8^{33}$

\section{MATERIALY MÉTODOS}

El presente estudio se realizó en el Servicio de Traumatología del Hospital Daniel Alcides Carrión Essalud- Tacna. Se revisaron las historias clínicas de pacientes mayores de 60 años de edad, con diagnóstico de fracturas de cadera, en el periodo comprendido entre enero del 2003 hasta diciembre del 2007. Población: Totalidad de historias clínicas de pacientes con diagnóstico de fractura del extremo proximal del fémur en el periodo de estudio. Muestra: no se consideró un tamaño muestral, ya que se incluyó a todos los integrantes de la población que cumplieron los criterios de selección.

Tipo de investigación Es un estudio descriptivo y retrospectivo.

Se investigó una serie de factores del estado de salud previo del paciente, así como la estancia hospitalaria que podrían influir en la morbilidad y mortalidad de la fractura del extremo proximal del fémur como: sexo, edad, patologías previas, el estado físico de acuerdo con la escala de valoración de la American Society of Anesthesiology (ASA), deambulación, el tipo de fractura, tratamiento utilizado, tipo de anestesia, estancia hospitalaria previa y posterior a la fractura. Estos datos se obtuvieron de las historias clínicas revisadas. Posteriormente se obtuvo información sobre complicaciones médicas y/o quirúrgicas relacionadas con la fractura de cadera. Luego se indagó si el paciente falleció durante el año posterior a la fractura.

\section{RESULTADOS}

Se produjeron un total de 58 casos, de los cuales el $70,69 \%$ fueron mujeres y $29,31 \%$ varones. La edad promedio de los varones afectados fue de 80,6 años, y en las mujeres de 78,7 años. En los pacientes mayores de 80 años se encuentra una mortalidad de $71,42 \%$ y en menores de 80 años se encontró una mortalidad de $28,58 \%$.

En la patología previa en los pacientes predominó la hipertensión arterial en $70,69 \%$, con demencia senil en $28,86 \%$, diabetes mellitus en $22,41 \%$, Enfermedad Pulmonar Obstructiva Crónica en 18,97\%, y osteoporosis en $17,24 \%$. Otras patologías menos frecuentes fueron hiperplasia benigna de próstata, 
insuficiencia renal crónica terminal, desnutrición, valvulopatías, enfermedad de Parkinson, entre otras.

El nivel de marcha de los pacientes previo a la fractura de cadera, se encontró un nivel de independencia en el $72,41 \%$ de casos y el $20,09 \%$ de los pacientes deambulaba con ayuda, sobre todo con apoyo familiar.

El $6,90 \%$ de ellos no deambulaba por las patologias preexistentes.

El $34,48 \%$ de pacientes no contenía los esfinteres antes de la caída de la fractura del extremo proximal del fémur, y el $65,52 \%$ sí contenían esfinteres.

El $53,45 \%$ se produjeron en el lado izquierdo y el $46,55 \%$ en el derecho. Predominó la fractura intertrocantérea en 39 casos $(67,24 \%)$, seguida de las fracturas a nivel de cuello $(32,76 \%)$. En las fracturas intertrocantéreas predominó el grado II de Tronzo, en las de cuello, el grado III de Garden.

Fallecieron 5 de los 31 que sufrieron fracturas intertrocantéricas, representando $16,13 \%$, que fue mayor que los pacientes fallecidos que sufrieron fractura de cuello de fémur, que fueron 2 del total de 27 representando el $7,41 \%$.

En su mayor parte los pacientes tuvieron un grado ASA II $(56,90 \%)$ o ASA III $(41,38 \%)$, con solo un paciente con ASAI.

En la fractura intertrocantérica se empleó sobre todo la reducción abierta con fijación interna (RAFI; 92,31\%). En las fracturas de cuello en un caso se empleó tratamiento médico con tracción, y en $84.21 \%$ colocación de prótesis parcial, con técnica de Gilderstone en $10,53 \%$.

En cuanto a la duración de la estancia preoperatoria, fue en promedio de 5 a 7 días, y la estancia postoperatoria estuvo entre 8 y 9 días en todas las técnicas quirúrgicas.

Se presentaron complicaciones en $41,38 \%$ de casos, de los cuales las complicaciones fueron médicas en $83,33 \%$ de casos, predominando la infección de tracto urinario $(35 \%)$, la producción de escaras de decúbito $(30 \%)$ o la neumonía $(25 \%)$. Entre las complicaciones quirúrgicas $(45,83 \%)$, se presentó con más frecuencia la infección de herida operatoria $(45.45 \%)$.

Se produjeron en total 7 muertes, que constituyen una mortalidad de $12,07 \%$.

Las causas de mortalidad fueron principalmente la sepsis $(42,86 \%)$, el infarto de miocardio o la neumonía $(28,57 \%)$.

\section{DISCUSIÓNY COMENTARIOS}

Dentro del conjunto de patologías que se presentaron como antecedente en el estudio, solo se encontró relación con la mortalidad para el accidente cerebrovascular que estuvo presente en el 14,29\% de los pacientes fallecidos y en ningún paciente que no falleció con una diferencia significativa del 0,01.De los pacientes que presentaron incontinencia urinaria, 4 fallecieron $(57,14 \%)$, no muestra diferencia significativa con los que no fallecieron.

En nuestro estudio no encontramos relación de la mortalidad respecto a los diferentes tipos de fractura de cadera, aunque se aprecia una mayor tasa de mortalidad para las fracturas intertrocantéricas del 16,13\% seguidas de cuello femoral $7,41 \%$. Se encontró una tasa de mortalidad de la fractura intertrocantérica parecida al $12,5 \%$ encontrada por Bellido, así como también el $6,9 \%$ de mortalidad para las fracturas del cuello femoral.

En algunos estudios se encuentra mayor mortalidad de las fracturas intertrocantéricas; se caracterizan por presentarse en pacientes 10 a 12 años más viejos que los pacientes con fractura de cuello de fémur, que son pacientes de mayor edad y, por consiguiente, con mayor riesgo de tener patologia de base 41 .

Se encontró una mayor tasa de mortalidad para el riesgo anestésico grado III $(71,43 \%)$ respecto a los grados ASA II $(28,57 \%)$, aunque no resultó una relación significativa. Los diferentes autores también obtienen mayores tasas de mortalidad para los grados ASAS III y IV. 21,42 .

En cuanto a la duración de la estancia preoperatoria, fue en promedio de 5 a 7 días. En el presente estudio no se encontró diferencia significativa en cuanto a mortalidad respecto al tiempo de demora preoperatorio.

Hay varios artículos que abordan este parámetro tratando de correlacionarlo con mortalidad, tratando de demostrar que un tratamiento precoz conlleva una mortalidad menor pero es difícil de comprobarlo, ya que el retraso en la intervención puede ser debida a múltiples causas.

Algunos opinan que se debería intervenir a los pacientes antes de las 24 horas 43,35 , ó 48 horas 44,25 y que más de tres días de retraso aumentaría la mortalidad 25,43.

Sin embargo, existirá un grupo de pacientes que se beneficiarán del retraso inferior de una semana 14 serían aquellos con patologías previas importantes 3 ó mas, el retraso nos permitirá una estabilización previa a la cirugía y estar en mejores condiciones posibles.

La tendencia actual viene resumida por Munuera 14. Si el paciente presenta más de 2 problemas médicos, es mejor retrasar la cirugía de la fractura de cadera hasta máximo de 5 días con el fin de operarlo en el mejor estado general posible. Por el contrario, si el paciente 
presenta menos de 2 problemas médicos, es mejor intervenirlo en las primeras 24 horas 44 .

La estancia hospitalaria postoperatoria estuvo entre 8 y 9 días en todas las técnicas quirúrgicas, y no se encontró diferencia significativa relacionada con el parámetro mortalidad

Los pacientes presentaron complicaciones médicas en $83,33 \%$ de casos, predominando la infección de tracto urinario (35\%), asi también Bellido encontró que la infección urinaria era la más frecuente, encontrado una incidencia de $65 \% 18$.

La producción de escaras de decúbito estuvo presente en el $30 \%$ de los pacientes, que coincide con la incidencia encontrada en diferentes estudios 18,21.

Así también se encontró que la neumonía estuvo presente en el $25 \%$ de los casos, es mayor a la encontrada en $12,93 \%$. La complicación más frecuente en otros trabajos fue la neumonía $26,31,32,38$.

\section{CONCLUSIONES}

1. La tasa de mortalidad después de una fractura del tercio del extremo proximal del fémur fue de $12,07 \%$, siendo la causa más frecuente la sepsis $(42,84 \%)$.

2. La tasa de complicaciones después de una fractura del tercio del extremo proximal del fémur fue de $41,38 \%$, siendo las complicaciones médicas las más frecuentes que las quirúrgicas.

3. Dentro de las características de los pacientes con fractura del tercio del extremo proximal del fémur en el Hospital Daniel Alcides Carrión está el promedio de edad que fue de 82 años, predominando el sexo femenino $(70,69 \%)$. La mayoría de los pacientes presentaron una marcha independiente antes de presentar la fractura de cadera, el $34.48 \%$ sufría de incontinencia urinaria. La hipertensión arterial predominó como antecedente de morbilidad (70,69\%). Predominó la fractura intertrocantérica $(67,24 \%)$ del grado II de Tronzo; la mayoría de los pacientes presentó riesgo anestésico de II grado $(56,90 \%)$. El tratamiento quirúrgico fue el más empleado, haciendo mayor uso de la anestesia general endovenosa, siendo el promedio del tiempo operatorio diferente en cada técnica quirúrgica.

4. Los factores asociados al desarrollo de complicaciones son la presencia del antecedente de diabetes, que se encontró en $45,83 \%$ de pacientes con complicaciones y $5,88 \%$ sin complicaciones. Otro factor fue la presencia de demencia senil $(41,67 \%$ en las complicaciones y $14,71 \% \sin$ complicaciones). Así como la postración de los pacientes fue antecedente en $16,67 \%$ de complicaciones y en ningún caso no complicado; otro factor fue la falta de control de esfinteres que se dio en la mitad de casos complicados y en el $23,53 \%$ de no complicados. Entre los factores de riesgo asociados a la mortalidad, tuvimos al antecedente de ACV (14,29\%) entre fallecidos, ausente entre los no fallecidos), y la falta de deambulación $(28,57 \%$ entre fallecidos y $3,92 \%$ entre no fallecidos).

\section{REFERENCIAS BIBLIOGRAFÍA}

BUCHOLZ, Robert W.; HECKMAN, JAMES D.; COURT-BROWN, CHARLES M. ROCKWOOD \&GREENS, Fractures in Adults. 6th edition. Copyright 2006 Lippincott Williams \& Wilkins

Canale S., T. Campbell. 2004. Cirugia Ortopédica. $10^{\circ}$ edición. 4 vol.

BERGLUND-RÖDEN M; SWIESTRA BA, Wingstrand $\mathrm{H}$, Thorngren KG. Prospective comparison of hip fracture treatment, 856 cases followed four months in the Netherlands and Sweden. Acta Ortop Scand 1994;65: 287-94.

MUNUERA, LUIS. Osteoporosis y fracturas. Ed Masson S.A, 2000, pag 4- 156.

BELLIDO, P. Análisis de las caracteristicas de los pacientes geriátricos con fractura del tercio proximal de fémur y la mortalidad en el primer año posterior a la fractura en el Servicio de Ortopedia y Traumatología del Hospital Central de la Fuerza Aérea del Perú 1994 1999. Tesis para optar Titulo Profesional presentado en la Universidad Nacional de San Agustín en el año 2000.

SOTORRES SENTANDREU, J. Morbilidad $y$ mortalidad en pacientes con fractura de cadera. Estudio Prospectivo. Trabajo para optar el grado de doctor en medicina y cirugía por la Universidad de Valencia. 2003

MEZA. E. Tesis. Fractura de cadera en ancianos. Universidad Nacional de San Agustín Arequipa, 1972.

MIRAVAL NIÑO DE GUZMÁN, T. "Fractura de cadera a trauma minimo en mayores de 50 años: morbimortalidad y pronóstico funcional". Revista Peruana de Reumatologia. 2001, Volumen 7, N², Lima.

COLLAZO ÁlVAREZ HIRALIO. "Morbimortalidad por fractura de cadera". Rev. Cubana Ortoped Traumatol. 2000; 14(1-2) 21-5.

BUSTAMANTE RAMÍREZ, RORY. Evaluación del tratamiento del tercio proximal de Oct 1996, Universidad Nacional de San Agustín.

MORELL, J; COLLAZO ÁlVAREZ, O'REILLY. Hip Fractures 1995-2005.

ESTÉVEZ LEZCANO LM, CARBAJALES LEÓN 
AI, GUTIÉRREZ BLANCO M, CARBAJALES LEÓN EB. "Evaluación del tratamiento quirúrgico de urgencia en fracturas de cadera en el período de enero/1996 a enero/1999". Rev. Arch Méd Camagüey. [serie en Internet] 2002 [citado 20 Ene 2008]; 6 (Supl 4): [aprox. 8 p.].

KENZORA JE, MCCARTHY RE, LOWELL JD, SLEDGE CB. "Hip fracture mortality, relation to age, treatment, preoperative illness, time of surgery and complications. Clin Orthop 1984; 186: 45-56.

Lyons AR. Clinical outcomes and treatment of hip fractures". Am J Med 1997; 103(2A): 51S-63S.

BROOS PL, VAN HAAFTEN KI, STAPPAERTS KH, GRUWEZ JA. "Hip fractures in the elderly.Mortality, functional results and social readaptation". Int Surg. 1989 Jul-Sep;74(3):191-4.
PARKER MJ, PALMER CR. "Prediction of rehabilitation after hip fracture". Age and Aging, 1995;24:96-98.

RICHMOND J, AHARONOFF GB, ZUCKERMAN JD, KOVAL KJ. "Mortality risk after hip fracture". J Orthop Trauma, 2003 Jan;17(1):53-6.

BREDAHL C. et al. "Mortality after hip fracture: results of operation within $12 \mathrm{~h}$ of admission". Injury, 1992; 23 (2): $83-6$.

KENZORA JE, et al. "Hip fracture mortality. Relation to age, treatment, preoperative illness, time of surgery, and complications". Clin Orthop, 1984 Jun, 186; 45-56. 